\title{
Sulla posizione \\ dell'asse di rotazione della terra rispetto all'asse di figura (*)
}

\author{
(del prof. Emanuele Fergora, a Napoli).
}

R semiasse maggiore ed $\varepsilon$ l'eccentricità, e supponiamo che la retta intorno alla quale si esegue la rotazione diurna non coincida con l'asse minore dell'ellissoide, ma sia un altro semidiametro, che comprenda con l'asse di figura un piccolo angolo ø; ciò che equivale a supporre l'asse principale minimo di inerzia non coincidente con l'asse di figura. Chiameremo meridiano geometrico qualunque sezione prodotta nello sferoide da un piano che passa per l'asse di figura, ed equatore geometrico la sezione che si ottiene dal piano condotto pel centro della terra perpendicolarmente all'asse medesimo.

In questa ipotesi sulla posizione dell'asse di rotazione, il meridiano astronomico di un qualsivoglia punto della superficie terrestre è il piano condotto per la normale parallelamente all'asse di rotazione; ed il meridiano geografico è il luogo dei punti in cui i meridiani astronomici sono fra loro paralleli. Il parallelo geografico di un punto qualunque è il luogo dei punti in cui le normali formano un angolo costante con l'asse di rotazione; ed in particolare l'equatore geografico è il luogo dei punti in cui le normali hanno direzioni perpendicolari all'asse di rotazione.

Da questi principii si desumono le seguenti proposizioni:

(*) Da una Memoria presentata all'Accademia di Scienze fisiche e matematiche, della Società Reale di Napoli, addi 7 maggio, 1874. 
1. Solamente i punti dell' equatore geometrico, e quelli del meridiano geometrico in cui giace l'asse di rotazione, hanno il meridiano astronomico che passa pure per l'asse di rotazione.

2. I meridiani geografici sono curve piane, e passano tutti per i due estremi di un diametro situato in un medesimo piano con l'asse di figura e quello di rotazione. Nella ellisse segnata da questo piano sulla superficie terrestre, la retta sulla quale s'incontrano tutti i meridiani geografici è il diametro conjugato alla direzione perpendicolare all'asse di rotazione; e gli estremi di questa retta sono i soli punti della superficie terrestre, dove la rotazione diurna sembra farsi intorno alla verticale.

Quindi chiamando $\varpi^{\prime}$ l'angolo che la comune sezione di tutti i piani dei meridiani geografici forma con l'asse di rotazione, sarà $\operatorname{tg} \omega^{\prime}=\frac{\operatorname{tg} \omega}{1-\varepsilon^{2}}$, donde si ricava $\varpi^{\prime}-\sigma=11^{\prime} 30^{\prime \prime} \cdot 65 \operatorname{sen} 2 \sigma+1^{\prime \prime} \cdot 16 \operatorname{sen} 4 \sigma+$ ecc.

3. Preso per primo meridiano il piano condotto per l'asse di figura e l'asse di rotazione, si chiami $v$ la longitudine di un qualunque punto, contato verso Ovest, e si dinotino con $I$ l'angolo che la sezione comune dei due meridiani, astronomico e geografico di quel punto, forma con l'asse di rotazione, e con $\sigma$ l'angolo diedro compreso fra i medesimi piani. È chiaro che, conoscendo $I$ e $\sigma$, rimane compiutamente determinata la posizione del meridiano geografico. Ora queste due quantità si possono ottenere dalle due equazioni

$$
\operatorname{tg} I=\operatorname{cotg} \varpi \sec v, \quad \sigma=\frac{\operatorname{sen} \nu}{\operatorname{sen} E \cos \varpi}\left(1-\operatorname{sen}^{2} \varpi \operatorname{sen}^{2} v\right)\left(\omega^{\prime}-\varpi\right)
$$

dove

$$
\cos E=\operatorname{sen} \omega \operatorname{sen} v .
$$

Da ciò si deduce facilmente la posizione della traccia orizzontale del meridiano geografico, poichè chiamando $\tau$ l'angolo che essa forma con la traccia orizzontale del meridiano astronomico, e $\phi$ la colatitudine, si ha

$$
\operatorname{tg} \tau=\cos (I-\phi) \operatorname{tg} \sigma .
$$

4. Gli assi maggiori di tutti i meridiani geografici si trovano sull'equatore geometrico e sono eguali all'asse maggiore dell'ellissoide. Gli assi minori invece stanno sopra una superficie conica, che ha il vertice al centro della terra, e passa per l'asse di figura e l'asse di rotazione. La sezione fatta in questo cono dal piano tangente all'estremo dell'asse di figura è un cerchio, che ha il centro nel piano del primo meridiano. Il meridiano geo- 
grafico di massima eccentricità è quello i cui punti hanno la longitudino astronomica eguale a zero; ed il meridiano geografico di minima eccentricita è quello in cui la longitudine astronomica di un punto qualunque è $90^{\circ}$.

5. Generalmente parlando i paralleli non sono curve piane, ma projettati dal centro della terra sul piano tangente all'estremo dell'asse di rotazione vi producono ellissi simili e similmente poste, che hanno il centro comune nel punto dove il suddetto piano è incontrato dal diametro pel quale passano tutti i meridiani geografici. L'asse minore di ciascuna di queste ellissi è situato nel piano del primo meridiano.

11 solo parallelo situato in un piano è quello di colatitudine $90^{\circ}$, cioè l'equatore geografico, ed è determinato dal piano diametrale conjugato all'asse di rotazione. Il suo asse minore trovasi nel primo meridiano.

Si puo trovare l'angolo $c$ compreso fra il meridiano geografico ed il parallelo, condotti per un medesimo punto, mediante l'equazione

$$
\iota=90^{\circ}-\pi \cdot \varepsilon^{2}[2 \operatorname{sen} \phi \operatorname{sen} v+\operatorname{sen} \omega \cos \phi \operatorname{sen} 2 v] \text {. }
$$

6. Un arco del meridiano astronomico di un punto compreso fra due paralleli qualunque, e l'arco del meridiano geografico del medesimo punto compreso fra gli stessi paralleli, sono fra loro eguali, a meno dei termini di $6^{\circ}$ grado rispetto all' eccentricità $\varepsilon$ ed al seno dell'angolo $\varpi$.

7. Ritenute le precedenti significazioni per $a, \varepsilon$ ed $\varpi$, sieno inoltre:

$s_{1}-s$ la lunghezza di un arco di meridiano geografico,

$\gamma_{1}$ e $\phi$ le colatitudini dei suoi estremi,

$l \quad$ la sua longitudine da un meridiano preso ad arbitrio, per esempio da Greenwich,

$v \quad$ la longitudine di Greenwich dal primo meridiano, cioè quello in cui si trova l'asse di figura;

si avrà fra tutte queste quantità la seguente equazione, a meno dei termini di grado superiore al $6^{\circ}$ rispetto ad $\varepsilon$ e sen $\varpi$,

$\left[(A+B) \operatorname{sen}^{2}(v+l)-2 B\right] \cos 2 \omega+2 C \cos (v+l) \operatorname{sen} 2 \sigma=(A+B) \operatorname{sen}^{2}(v+l)-2(A-D)$, dove $A, B, C, D$ sono ricavate dalle seguenti formole:

$$
\begin{array}{ll}
A=f\left(\Phi_{1}-\phi\right)+g \Phi_{1}+i \Phi_{2} & B=m \Phi_{1}+n \Phi_{2} \\
C=m \Psi_{1}+i \Psi_{2} & D=p\left(\Phi_{1}-\phi\right)+q \Phi_{1}+r \Phi_{2}+t \Phi_{3}-\frac{s_{1}-s}{a}
\end{array}
$$




$$
\begin{array}{ccc}
f=\frac{1}{4} \varepsilon^{2}\left(1+\frac{11}{8} \varepsilon^{2}\right) & g=-\frac{7}{16} \varepsilon^{4} & i=\frac{15}{64} \varepsilon^{4} \\
m=-\frac{3}{4} \varepsilon^{2}\left(1+\frac{1}{4} \varepsilon^{2}+\frac{173}{184} \varepsilon^{4}\right) & n=\frac{15}{32} \varepsilon^{4} & p=1+\frac{19}{64} \varepsilon^{1}-\frac{5}{256} \varepsilon^{6} \\
q=-\frac{7}{16} \varepsilon^{4}\left(1+\frac{4}{7} \varepsilon^{2}\right) \quad r=-\frac{15}{128} \varepsilon^{1}\left(1-\frac{3}{4} \varepsilon^{2}\right) & t=\frac{35}{1536} \varepsilon^{6} \\
\Phi_{1}=\operatorname{sen}\left(\phi_{1}-\zeta\right) \cos \left(\phi_{1}+\phi\right) & \Psi_{1}=\operatorname{sen}\left(\phi_{1}-\phi\right) \operatorname{sen}\left(\phi_{1}+\phi\right) \\
\Phi_{2}=\operatorname{sen} 2\left(\phi_{1}-\phi\right) \cos 2\left(\phi_{1}+\phi\right) & \Phi_{2}=\operatorname{sen} 2\left(\phi_{1}-\phi\right) \operatorname{sen} 2\left(\phi_{1}+\phi\right) \\
\Phi_{3}=\operatorname{sen} 3\left(\phi_{1}-\phi\right) \cos 3\left(\phi_{1}+\phi\right) .
\end{array}
$$

Ammettendo che nella precedente relazione si possano introdurre per $s_{1}-s$ i valori ottenuti con misure geodetiche per varii cerchi di meridiano, si potranno formare diverse equazioni contenenti le due incognite $v$ ed $\varpi$, e si avranno quindi i valori di queste incognite. Cosi, per esempio, ritenendo per $a$ ed $\varepsilon \dot{i}$ valori trovati da Bessel $\left(^{*}\right)$, e facendo uso dei seguenti dati numerici $\left(^{* *}\right)$ desunti dalle maggiori operazioni geodetiche finora eseguite, si ottengono le infrascritte nove equazioni di condizione per trovare le incognite $v$ ed $\varpi$.

I. Arco Anglo-francese. Saxavord-Formentera

$$
\phi=29^{\circ} 10^{\prime} 22^{\prime \prime} \cdot 79 \quad \zeta_{1}=51^{\circ} 20^{\prime} 6^{\prime \prime} \cdot 83 \quad l=-0^{\circ} 30^{\prime} \quad s_{1}-s=1264645^{\mathrm{x}} \cdot 92 ;
$$

II. Arco Russo. Fuglences-Staro Nekrassowka

$\phi=19^{\circ} 19^{\prime} 48^{\prime \prime} .77 \quad \phi_{1}=44^{\circ} 39^{\prime} 57^{\prime \prime} .06 \quad l=-26^{\circ} 40^{\prime} \quad s_{1}-s=1447786^{\mathrm{T}} \cdot 783 ;$

III. Arco Indiano $\left(2^{\circ}\right)$. Kaliana-Punnœ

$\phi=60^{\circ} 29^{\prime} 11^{\prime \prime} \cdot 678 \quad \phi_{1}=81^{\circ} 50^{\prime} 28^{\prime \prime} \cdot 868 \quad l=-77^{\circ} 40^{\prime} \quad s_{1}-s=1212885^{\mathrm{T}} \cdot 34 ;$

IV. Arco del Capo di Buona Speranza. N. End-Cape Point

$\grave{\phi}=119^{\circ} 44^{\prime} 17^{\prime \prime} \cdot 66 \quad \phi_{1}=124^{\circ} 21^{\prime} 6^{\prime \prime} \cdot 26 \quad l=-18^{\circ} 30^{\prime} \quad s_{1}-s=262470^{\mathrm{T}} \cdot 39 ;$

(*) Astronomische Nachrichten, $n{ }^{\circ} 438$.

(**) Ordnance Trigonometrical Survey of Great Britain and Ireland. - Account of the Observations and Calculations of the principal triangulation. London, 1858, pag. 759.

Comparisons of the standards of Lenght, made at the Ordnance Survey Office, Southampton, by Cap. A. R. Clarcke, under the direction of Col. Sir H. James. London, 1866, pag. 282-283.

Annali di Matematica, tomo VI. 
V. Arco Peruviano. Cotchesqui-Tarqui

$$
\phi=89^{\circ} 57^{\prime} 28^{\prime \prime} \cdot 613 \quad \phi_{1}=93^{\circ} 4^{\prime} 32^{\prime \prime} \cdot 068 \quad l=+79^{\circ} 0^{\prime} \quad s_{1}-s=176875^{\mathrm{r}} \cdot 5
$$

VI. Arco Annoverese. Altona-Gottinga

$$
\phi=36^{\circ} 27^{\prime} 14^{\prime \prime} \cdot 73 \quad \gamma_{1}=38^{\circ} 23^{\prime} 12^{\prime \prime} \cdot 15 \quad l=-9^{\circ} 57^{\prime} \quad s_{1}-s=115163^{\mathrm{x}} \cdot 725 ;
$$

VII. Arco Danese Lysabbel-Lauenburg

$$
\phi=35^{\circ} 5^{\prime} 49^{\prime \prime} \cdot 648 \quad \phi_{4}=36^{\circ} 37^{\prime} 42^{\prime \prime} .9 \mathrm{a} 4 \quad l=-10^{\circ} 30^{\prime} \quad s_{1}-s=87436^{\mathrm{r}} \cdot 538 ;
$$

VIII. Arco Prussiano. Memel-Trunz

$$
\phi=34^{\circ} 16^{\prime} 19^{\prime \prime} .554 \quad \phi_{1}=35^{\circ} 46^{\prime} 48^{\prime \prime} .534 \quad l=-20^{\circ} 30^{\prime} \quad s_{1}-s=86176^{\mathrm{x}} \cdot 975 ;
$$

IX. Arco Indiano $\left(1^{\circ}\right)$. Paudree-Trivandeporum

$$
\begin{aligned}
\phi= & 76^{\circ} 40^{\prime} 10^{\prime \prime} .982 \quad \phi_{1}=78^{\circ} 15^{\prime} \gamma^{\prime \prime} \cdot 410 \quad l=-79^{\circ} v^{\prime} \quad s_{1}-s=89815^{\mathrm{T}} \cdot 45 \\
\text { I } \quad 0= & {\left[+0.94135 \operatorname{sen}^{2}\left(v-0^{\circ} 30^{\prime}\right)-0.59624\right] \cos 2 \sigma+} \\
& +3.73600 \cos \left(v-0^{\circ} 30^{\prime}\right) \operatorname{sen} 2 \varpi-0.94135 \operatorname{sen}^{2}\left(v-0^{\circ} 30^{\prime}\right)+0.65686 \\
\text { II } \quad 0= & {\left[+1.66678 \operatorname{sen}^{2}\left(v-26^{\circ} 40^{\prime}\right)-1.86160\right] \cos 2 \varpi+} \\
& +3.86966 \cos \left(v-26^{\circ} 40^{\prime}\right) \operatorname{sen} 2 \varpi-1.66678 \operatorname{sen}^{2}\left(v-26^{\circ} 40^{\prime}\right)+2.01662
\end{aligned}
$$

III $0=\left[-0.80663 \operatorname{sen}^{2}\left(v-77^{\circ} 40^{\prime}\right)+2.88330\right] \cos 2 \varpi+$

$$
+2.21806 \cos \left(v-77^{\circ} 40^{\prime}\right) \operatorname{sen} 2 \omega+0.80663 \operatorname{sen}^{2}\left(v-77^{\circ} 40^{\prime}\right)-2.81652
$$

IV $0=\left[-0.04306 \operatorname{sen}^{2}\left(v-18^{\circ} 30^{\prime}\right)+0.35660\right] \cos 2 \omega-$

$$
-0.72290 \cos \left(v-18^{\circ} 30^{\prime}\right) \operatorname{sen} 2 \sigma+0.04306 \operatorname{sen}^{2}\left(v-18^{\circ} 30^{\prime}\right)-0.34512
$$

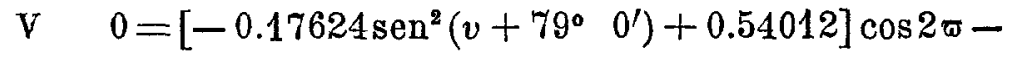

$-0.02862 \cos \left(v+79^{\circ} 0^{\prime}\right) \operatorname{sen} 2 \pi+0.17624 \operatorname{sen}^{2}\left(v+79^{\circ} 0^{\prime}\right)-0.52840$

VI $0=\left[+0.10305 \operatorname{sen}^{2}\left(v-9^{\circ} 5 \gamma^{\prime}\right)-0.11688\right] \cos 2 \sigma+$ $+0.34138 \cos \left(v-9^{\circ} 57^{\prime}\right) \operatorname{sen} 2 \sigma-0.10305 \operatorname{sen}^{2}\left(v-9^{\circ} 57^{\prime}\right)+0.13756$

VII $0=\left[+0.08552 \operatorname{sen}^{2}\left(v-10^{\circ} 30^{\prime}\right)-0.08224\right] \cos 2 w+$ $+0.25516 \cos \left(v-10^{\circ} 30^{\prime}\right) \operatorname{sen} 2 v-0.08552 \operatorname{sen}^{2}\left(v-10^{\circ} 30^{\prime}\right)+0.07350$

VIII $0=\left[+0.08791 \operatorname{sen}^{2}\left(v-20^{\circ} 30^{\prime}\right)-0.08836\right] \cos 2 \pi+$ $+0.24878 \cos \left(v-20^{\circ} 30^{\prime}\right) \operatorname{sen} 2 \varpi-0.08791 \operatorname{sen}^{2}\left(v-20^{\circ} 30^{\prime}\right)+0.12002$ 
IX $\quad 0=\left[-0.07731 \operatorname{sen}^{2}\left(v-79^{\circ} 0^{\prime}\right)+0.24934\right] \cos 2 \sigma+$

$+0.11650 \cos \left(v-79^{\circ} 0^{\prime}\right) \operatorname{sen} 2 \sigma+0.07731 \operatorname{sen}^{2}\left(v-79^{\circ} 0^{\prime}\right)-0.24262$.

I valori di $v$ e $\varpi$, che rendono minima la somma dei quadrati dei secondi membri di queste equazioni sono

$$
v=239^{\circ} 31^{\prime} \quad \varpi=1^{\circ} 8^{\prime} ;
$$

quindi, secondo questi calcoli, uno degli estremi dell'asse di figura della terra avrebbe la longitudine $120^{\circ} 29^{\prime}$ Ovest da Greenwich, e la latitudine $88^{\circ} 52^{\prime}$ Nord.

Napoli, 12 maggio 1874. 